\title{
Computerized tomography and clinical correlation of stroke diagnosis in University of Port Harcourt Teaching Hospital
}

\author{
${ }^{1}$ Onubiyi Chidiogo Chiedozie Brown*, ${ }^{2}$ Nwankwo Nelson Chukwuemeka, ${ }^{3}$ Onwuchekwa Regina \\ Chinwe, ${ }^{4}$ Ray-Offor Ogheneochuko Deborah, ${ }^{5}$ Eweputanna Lisa Ifeyinwa \\ Department of radiology, University of Port-Harcourt teaching hospital, Port-Harcourt, Rivers State, Nigeria \\ ${ }^{*}$ Corresponding author's email: diogobrown@yahoo.com
}

\begin{abstract}
The burden of stroke is high and is not only attributable to its high mortality but also its consequent high morbidity. Wrong clinical diagnosis has a high significance for patient outcome. Thus the need for accurate stroke diagnosis. Certainty of stroke diagnosis is markedly enhanced by computed tomography (CT) which has remained the most important brain imaging test for stroke in an emergency. This study was done to confirm clinical diagnosis of stroke using CT as seen in University of Port Harcourt Teaching Hospital (UPTH) and to determine the varying presentations and other findings which could mimic stroke clinically. This was a prospective study of 203 subjects with clinical diagnosis of stroke who had CT of the brain performed during a 12month period (November 2012 to November 2013). A 2-slice helical CT was used to obtain images in the axial plane. Images were classified as normal or abnormal scans. Abnormal scans were analyzed as ischaemic infarcts, intracerebral haemorrhage, subarachnoid haemorrhage (SAH), subdural haematoma, abscess, neoplasm, meningitis, and cerebral atrophy. The clinical diagnosis, age, sex and time of presentation of the patients were recorded. Statistical analysis was done with the Statistical Package for the Social Sciences (SPSS) software version 20.0. Means were compared using Student's $t$ test. Pearson's correlation coefficient was used to correlate CT findings with clinical diagnosis, age, and sex. $P$ values $<0.05$ were considered significant. The age distribution of the study population ranged from 6-90 years with a mean of $58.3 \pm 14.9$ years. Males were predominant at $51.7 \%$ of the sample size. The clinical diagnosis of stroke was confirmed in $\mathbf{7 4 . 9 \%}$ of subjects while stroke mimics accounted for $13.3 \%$, the rest $(11.8 \%)$ were normal. This gave a misdiagnosis rate of $\mathbf{2 5 . 1 \%}$. Specific clinical diagnosis of stroke subtype (ischaemic or haemorrhagic) was correct in only $60 \%$ of cases. Despite a good rate of accurate clinical stroke diagnosis, a significant rate is misdiagnosed with potential for grave implication on patient morbidity. CT should be an essential aspect of stroke management.
\end{abstract}

Key words: Computed tomography, Cerebrovascular disease, Stroke, Ischaemic stroke, Haemorrhagic stroke, University of Port Harcourt Teaching Hospital.

\section{ABBREVIATIONS}

Computed tomography (CT).

University of Port Harcourt Teaching Hospital (UPTH)

Cerebrovascular disease (CVD)

Subarachnoid haemorrhage (SAH)

\section{INTRODUCTION}

Stroke is defined as sudden diminution or loss of consciousness, sensation, and voluntary motion caused by rupture or obstruction (as by a clot) of a blood vessel of the brain (Merriam - Webster 2014). It is a common 
cause of hospitalization, morbidity and mortality in our environment.Stroke is of two types; ischaemic and haemorrhagic. Haemorrhagic stroke has two subtypes; intra-cerebral haemorrhage and subarachnoid haemorrhage (CDC 2012)

Clinically, a definite diagnosis of either haemorrhagic stroke or ischaemic stroke may not be made in all patients (Runchey et al 2010). Imaging plays a crucial role in confirming, differentiating subtypes and so in maintaining, modifying or changing treatment of stroke.

Computed tomography (CT), readily differentiates the subtypes of stroke, excludes tumors, extra-axial lesions, infections and other stroke-like conditions. CT due to its increasing availability, speed, and patient tolerance, now plays a vital role in making the diagnosis, defining the pattern and enhancing management of strokes in our environment.

Non-enhanced CT scan is recommended by the American Heart Association as the initial modality of choice for stroke investigation (Adams et al 2007). Time is of essence in management of strokes and confirming the diagnosis contributes to improved prognosis. Magnetic Resonance Imaging, angiography and ultrasonography are all quite useful in stroke imaging. CT however allows "rapid triage of patients suspected of having experienced a stroke" (Hopyan et al 2010).

Clinical scoring has improved the diagnosis of stroke but it is advised to be used in the absence of appropriate imaging modality (Kolapo et al 2005). Clinically, certain findings may point towards ischaemic or hemorrhagic stroke but definite diagnosis in all patients cannot be guaranteed. Limitations of clinical stroke diagnosis has been established in various locations (Asefa et al 2010, Bamford 1992, Salawu et al 2009, Ogun et al 2001)

Stroke mimics which are certain conditions that can present with a clinical picture similar to that of stroke, have been reported (Libman et al 1995, Ogun et al 2000, Onwukeme et al 2008). They may include intracranial neoplasm, subdural hematomas, abscess, cerebral atrophy, post-ictal states, etcetera.

Therapy for both ischaemic and haemorrhagic stroke differ and may be detrimental if treatment for one is given for another. It is therefore important that stroke diagnosis and subtype are confirmed as this influences the course of management.

Stroke is the $3^{\text {rd }}$ leading cause of death in the western world ( Dawson and Walters 2006). It is known to have a high prevalence in Nigeria (Danesi et al 2007) It causes a lot of emotional, psychological, financial, social and economic burden on both patient and relatives and even on the health delivery system (Palmer et al 2005, Vila and Irimia 2004, Ingali 2004).

This study aims at ascertaining the clinical diagnosis of stroke in UPTH by means of computed tomography and excluding stroke mimics.

\section{MATERIALS AND METHODS}

\section{Study site}

This study was carried out in the radiology department of University of Port Harcourt Teaching Hospital (UPTH) in the South South geo-political zone of Nigeria over a period of 12 months; from November 2012 to November 2013. This hospital is a 500 bed facility that serves as a referral centre for Rivers State and the neighboring south- south states of the Niger Delta.

\section{Study Design}

The study was a cross-sectional, prospective and descrpitive study which utilized subjects referred to the department of radiology, UPTH for brain CT with clinical diagnosis of stroke. Utilized patients were recruited into the study after meeting the set criteria. Consent was sought with signatures on a form after counseling and confidentiality was assured. Subject's relatives appended their signatures in cases where the subjects were not able to do so.

Clinical diagnosis, age, sex, time of onset of symptoms and time of presentation to the hospital, as well as time between presentation to the hospital and CT were obtained from subjects' relatives and case notes using a structured interview form.

\section{Imaging Technique}

All the brain scans were done using a General Electric NX/1 Dual Slice Helical CT scanner. The patients were counseled where possible. They were then laid supine with head first into the gantry. Patient's head was steadied in the head holder. Scanogram was obtained, and then contiguous axial slices of $5 \mathrm{~mm}$ thickness and interval of non-enhanced scans were taken from the skull base to the vertex. This was followed by contrastenhanced scans for those in whom native scans were normal or showed infarcts or mass lesions except haemorrhage.

\section{Data Analysis}

Data analysis was done using Statistical Package for Social Sciences (SPSS) software (version 20.0) for windows. Results were presented as mean \pm standard deviation, percentages, tables and graphs as appropriate. Means were compared using Student's $t$ test. Pearson's correlation was used to assess the association between CT findings, socio demographic factors and clinical diagnosis. $P$ values less than or equal to 0.05 were considered statistically significant. 
92 J. Med. Med. Sci.

\section{RESULTS}

Table 1. CT findings of patients

\begin{tabular}{lc}
\hline CT Findings & Frequency (\%) \\
\hline Normal & $24(11.8)$ \\
Infarcts & $96(47.3)$ \\
Intraparenchymal bleed & $52(25.6)$ \\
Subarachnoid haemorrhage & $4(2.0)$ \\
Abscess & $6(3.0)$ \\
Neoplasm & $1(0.5)$ \\
Meningitis & $1(0.5)$ \\
Atrophy & $16(7.9)$ \\
Subdural haematoma & $3(1.5)$ \\
\hline Total & $\mathbf{2 0 3 ( 1 0 0 )}$ \\
\hline
\end{tabular}

Table 2. CT outcome of patients based on time of presentation

\begin{tabular}{|c|c|c|c|c|}
\hline \multirow[b]{2}{*}{ CT Finding } & \multicolumn{3}{|c|}{ Time of presentation } & \multirow{2}{*}{ Tota } \\
\hline & $<6$ hours & 6-24hours & $>24$ hours & \\
\hline Normal & 1 & 4 & 19 & 24 \\
\hline Stroke mimics & 0 & 4 & 23 & 27 \\
\hline Infarcts & 0 & 6 & 90 & 96 \\
\hline Haemorrhage & 0 & 11 & 45 & 56 \\
\hline Total & 1 & 25 & 177 & 203 \\
\hline
\end{tabular}

A total of 203 subjects were recruited into the study. The age range was 6-90 years with a mean of $58.3 \pm 14.9 y e a r s$. The age group $70 y e a r s$ and above had the highest frequency with $49(24.1 \%)$ subjects and age group of 20years and younger having the lowest with 1 subject $(0.5 \%)$.

Of the total number of subjects, $105(51.7 \%)$ were males and $98(48.3 \%)$ were females with a male to female ratio of 1.1:1. This was not statistically significant; $p=0.624$. There were more male subjects, $27(25 \%)$ in the $51-60$ age group while females were more, $26(26.5 \%)$ in the $61-70$ age group.

All patients had clinical diagnosis of stroke but only 43 $(21 \%)$ had clinical diagnosis of specific stroke subtype:23 $(53.5 \%)$ ischaemic and 20 (46.5\%) haemorrhagic.

On computed tomography scan, 24subjects (11.8\%) had normal CT diagnosis while 179 (88.2\%) had abnormal diagnosis.

Out of 203 patients, $152(74.9 \%)$ of the clinical diagnosis were confirmed as CVD on CT $(p=0.010)$ while
$24(11.8 \%)$ were normal and $27(13.3 \%)$ were stroke mimics $(p=0.544)$. The stroke mimics included cerebral atrophy, cerebral abscess, subdural haematoma, meningitis and intracranial neoplasm. A total misdiagnosis rate of $25.1 \%$ was noted.

Of the 152 confirmed cases of CVD on CT, ischaemic CVD was predominant with a frequency of $96(63.2 \%)$. Cerebral haemorrhages were noted in 56cases (36.8\%), comprising intraparenchymal haemorrhage 52 (34.2\%) and $\mathrm{SAH} 4(2.6 \%)$.For the specific clinical diagnosis of CVD subtypes, only $60 \%$ were so identified on CT ( $p=$ $0.010 ; r=0.287$ ), that is 15 out of 23 for ischaemic and 11 out of 20 for haemorrhagic. Three (3) cases of ischaemic CVD were misdiagnosed as haemorrhagic while an equal number of cases of haemorrhagic CVD were also misdiagnosed as ischaemic, giving a cumulative frequency of $6(14 \%)$ while the remaining 11 cases $(26 \%)$ were either normal or stroke mimics. 


\section{DISCUSSION}

Stroke is an important disease worldwide, constituting a big burden on the public health purse as well as on patients and their relations. Accurate stroke diagnosis and subtype confirmation therefore plays a big role in patient management and prognosis. CT has enhanced stroke management by confirming the diagnosis, differentiating stroke subtypes and excluding stroke mimics (Lee 1996). CT has become essential in management of stroke and is advocated as a first line investigation (Adams et al 2007, Wardlaw et al 2004) in a patient suspected of having had a stroke. This study emanated to evaluate the CT findings of clinically diagnosed stroke in UPTH.

A total of 203 subjects who were clinically diagnosed of having had a stroke were analyzed in this study. The mean age of the subjects was 58.3years. Asefa et al (2007) in a CT stroke study in Ethiopia, had a mean age of 50.6years while a study of stroke diagnosis with CT in Enugu by Onwukeme et al (2008) had a higher mean age at 53years. The mean ages of the aforementioned studies are slightly different from this study; both studies are however retrospective. Salawu et al (2009) in their prospective study of CT stroke profile and clinical scoring of 95 patients in Maiduguri, had a mean age of 55.4years. This is much closer to the mean age of this study.

In this study there was marginal male predominance as males accounted for $51.7 \%$ of the study population while females were $48.3 \%$ with a male to female ratio of $1 \cdot 1: 1$. This was not statistically significant. Sex distribution in the Salawu et al (2009) study showed a male predominance with a male to female ratio of $1.9: 1$. The study of "sex difference in stroke" in United States by Turtzo and McCullough (2008) as well as the El Zein et al (2007) study collaborated male predominance. The possible reason for the concordance in male predominance may be that the predisposing factors such as lifestyle, hypertension, diabetes mellitus, etcetera, affected males more. It may also be that males sought hospital treatment more. Onwukeme et al (2008) in Enugu even showed higher male predominance. The possible explanation may be that health seeking behavior amongst women in Port Harcourt is better than that of those in Enugu.

Ischaemic infarcts were the most observed in this study in $63.2 \%$ of subjects while Haemorrhagic CVD was seen in $36.8 \%$ of subjects.

This study agrees with $\mathrm{Ng}$ et al (1998), Sotaniemi et al (1990) and Vila and Irimia (2004) that ischaemic CVD was more predominant in both sexes but it was discovered that haemorrhagic CVD had a much higher predominance in men. This implies that gender variation has relatively no role in incidence of ischaemic stroke, or that males tend to suffer more from factors that predispose to haemorrhage.
Reliance on clinical diagnosis of stroke alone is not justifiable, especially where CT is readily available. Only $74.9 \%$ of the clinical diagnosis of the subjects in this study were confirmed on CT with a misdiagnosis rate of $25.1 \%$. This rate is even higher when narrowed to clinically diagnosed stroke subtypes with a correct diagnosis of $60 \%$ and misdiagnosis of $40 \%$. This finding of inadequacy of clinical diagnosis is in consonance with Salawu et al (2009) finding in a study in Maiduguri, where they compared clinical diagnosis of stroke with CT diagnosis, noting a misdiagnosis rate of $15 \%$. In a similar study to Salawu et al's, Asefa et al (2010) in Ethiopia reported a misdiagnosis rate of $30 \%$. Ogun et al (2001) in a study of 156 Nigerian patients to assess the frequency of misdiagnosis of stroke using CT, reported a high misdiagnosis rate of $44 \%$. All these point to widely agreed notion that clinical diagnosis of stroke is sometimes not reliable despite the use of complex clinical scoring modules.

Most patients $(79 \%)$ sent for CT had a diagnosis of CVD without specifying the subtype, also suggesting clinical limitation in differentiating ischaemic from hemorrhagic CVD. These rates are important to note considering that wrong therapy for the stroke subtype may be catastrophic for the patient as noted in some studies MAST-1 (1995).

A possible cause of wrong clinical diagnosis is due to stroke mimics which may present with similar clinical features to stroke. This study had a number of stroke mimics accounting for 27 (13.3\%). Ogun et al (2000) also collaborated this in their previous mentioned study reporting a similar percentage, $13.5 \%$, who could have benefitted from surgical treatment. Onwukeme et al (2008) in a study of stroke mimics in Enugu using CT even reported a higher stroke mimic rate of $23.2 \%$, noting that despite the use of clinical scoring systems in stroke diagnosis, that high rate of misdiagnosis has potential implications for adequate management of the patients. Another possible reason for inaccurate clinical diagnosis could be that due to high patient load, many patients may therefore not undergo detailed clinical assessment due to inadequate physicians.

Kolapo et al (2005) concluded that clinical diagnosis closely correlates with CT finding. This is at variance with this study finding. But Kolapo et al also stressed that despite their assertion, that clinical scoring should come into play only where CT is not available.

\section{CONCLUSION}

This study found that clinical diagnosis of stroke does not always correlate with CT diagnosis; this is even worse when narrowed to clinical diagnosis of specific stroke subtype, creating a high potential for poor patient morbidity. It is therefore pertinent that CT be incorporated in stroke management where possible. 
94 J. Med. Med. Sci.

\section{REFERENCES}

Adams HP Jr, del Zoppo G, Alberts MJ, Bhatt DL, Brass L, Furlan A, Grubb RL, Higashida RT, Jauch EC, Kidwell C, Lyden PD, Morgenstern LB, Qureshi AI, Rosenwasser RH, Scott PA, Wijdicks EFM (2007). Guidelines for the early management of adults with ischemic stroke: a guideline from the American Heart Association/American Stroke Association Stroke Council, Clinical Cardiology Council, Cardiovascular Radiology and Intervention Council, and the Atherosclerotic Peripheral Vascular Disease and Quality of Care Outcomes in Research Interdisciplinary Working Groups. Stroke 2007;38 (5):1655-1711.

Asefa G, Meseret SCT and Clinical Correlation Of Stroke Diagnosis, Pattern And Clinical Outcome Among Stroke Patients Visiting Tikur Anbessa Hospital. Ethiop Med J 2010; 48(2): 117-112.

Bamford J. (1992) Clinical Examination In Diagnosis And Sub classification Of Stroke. Lancet; 339: 400-402.

Danesi M, Okubadejo N, Ojini F (2007). Prevalence of stroke in an urban mixed income community in Lagos, Nigeria. Neuroepidemiology; 204:1-8.

Dawson J, Walters M (2006). New and Emerging Treatments for Stroke. British Medical Bulletin 77-78(1): 87-102.

El Zein AME, Bukhari EA, Homeida S, Adam I (2007). Stroke In CT Scan Department Of Khartoum Hospital, Sudan. Trop Doct; 37(4): 244-245.

Hopyan J, Ciarallo A, Dowlatshahi D, Howard P, John V, Yeung R Zhang L, Kim J, MacFarlane G, Lee T, Aviv RI (2010). Certainty of Stroke Diagnosis: Incremental Benefit with CT perfusion over noncontrast CT and CT Angiography. Radiology; 255: 142-153.

Ingall T (2004). Stroke : Incidence, Mortality, Morbidity and Risk. Journal of Insurance Medicine; 36:143-152.

Kolapo KO, Ogun SA, Danesi MA, Osalesi BS, Odusote KA (2005). Validation Study Of The Siriraj Stroke Score In African Nigerians And Evaluation Of The Discriminant Values Of Its Parameters. Stroke; 37: 1197-2000.

Lee DH (1996). Brain Imaging In Stroke. AJNR; 17: 1265-1266.

Libman RB, Wirkowski E, Alvir J, Rao TH (1995). Conditions That Mimic Stroke In The Emergency Department: Implications For Acute Stroke Trials. Arch Neurol; 52(11): 1119-1122.

Multicenter Acute Stroke Trial - Italy (MAST-1). Randomized Controlled Trial of Streptokinase, Aspirin And Combination Of Both In

Treatment of Acute Ischaemic Stroke. Lancet 1995; 346: 1509-1514.
National Institute of Neurological Disorders rt - PA Stroke Study Group. Tissue Plasminogen Activator for Acute Ischaemic Stroke. N Engl J Med 1995; 333:1581-1587.

Ogun SA, Oluwole O, Fatade B, Ojini F, Odusote KA, Ogunseyinde AO (2001). Accuracy of The Siriraj Stroke Score In Differentiating Cerebral Hemorrhage And Infarction In African Nigerians. Afr $\mathrm{J}$ Neurol Sci; 20: 21-26.

Ogun SA, Oluwole O, Ogunseyinde AO, Fatade B, Odusote KA (2000). Misdiagnosis of Stroke. A Computed Tomography Scan Study. West Afr J Med; 19(1): 19-22.

Onwukeme IO, Ezeala-Adikaibe BA, Ohaegbulam SC, Chikani MC, Amuta J, Uloh HN (2008). Stroke Mimics - A Study Of CT Images In Nigerian African Stroke Patients. J Neurol Sci (Turk); 25(3): 148154.

Palmer AJ, Valentine WJ, Roze S, Lammert M, Spiesser J, Gabriel S (2005). Overview of costs of stroke from published, incidence-based studies spanning 16 industrialised countries. Curr Med Res Opin; 21(1) : 19-26.

Runchey S, McGee S (2010). Does This Patient Have a Hemorrhagic Stroke? Clinical Findings Distinguishing Hemorrhagic Stroke From Ischaemic Stroke. JAMA;303(22):2280-2286.

Salawu F, Umar I, Danburam A (2009). Comparison Of Two Hospital Stroke Scores With Computed Tomography In Ascertaining Stroke Type Among Nigerians. Ann Afr Med; 8: 14-52.

Sotaniemi KA, Pyktinen J, Myllyia VV (1990). Correlation Of Clinical And Computed Tomographic Findings In Stroke Patients. Stroke; 21: $1562-1566$

Stroke: Definition of stroke. www.merriam-webster.com/medical/stroke. Accessed on April 30, 2014.

Stroke:Types. www.cdc.gov/stroke/types_of_stroke.htm. Accessed on 24 May, 2012.

Turtzo LC, McCullough LD (2008). Sex Differences In stroke. Cerebrovasc Dis; 26: 462-474.

Vila EM, Irimia P (2004). The cost of stroke. Cerebrovasc Dis; 17: 1249.

Wardlaw JM, Keir SI, Seymour J, Lewis S, Sandercock PA, Dennis MS Cairns J (2004). What Is The Best Imaging Strategy For Acute Stroke? Health Technol Assess; 8: 1-180. 